Espacio y Desarrollo N 34, 2019, pp. 175-200 (ISSN 1016-9148) https://doi.org/10.18800/espacioydesarrollo.201902.008

\title{
¿EXISTE RIESGO DE CAÍDA DE ÁRBOLES DE SCHINUS MOLLE POR LA PRESENCIA DE TUMORES EN LA CIUDAD DE LIMA?
}

\author{
Ximena del Pilar Colán de la Vega \\ https://orcid.org/0000-0002-7450-0150 \\ Facultad de Ciencias Forestales, Universidad Nacional Agraria La Molina \\ ximenacdlv@gmail.com \\ José Alberto Cipra Rodriguez \\ https://orcid.org/0000-0002-7816-5839 \\ Facultad de Ciencias Forestales, Universidad Nacional Agraria La Molina \\ jaciprarodriguez@gmail.com \\ Angel Eduardo Montoya Yanavilca \\ https://orcid.org/0000-0001-9100-5787 \\ Facultad de Ciencias Forestales, Universidad Nacional Agraria La Molina \\ angelemonya@gmail.com \\ Julio Jeanpierre Adriano Reyes \\ https://orcid.org/0000-0002-6302-7660 \\ Facultad de Ciencias Forestales, Universidad Nacional Agraria La Molina \\ jpadrianor@gmail.com \\ Maricel Jadith Móstiga Rodríguez \\ https://orcid.org/0000-0002-2604-463X \\ Facultad de Ciencias Forestales, Universidad Nacional Agraria La Molina \\ mmostiga@lamolina.edu.pe
}

Fecha de recepción: 26/06/2019 Fecha de aceptación: 18/05/2020

\section{RESUMEN}

La Molina es uno de los distritos con mayor cantidad de áreas verdes y conciencia ecológica de la ciudad de Lima. La Universidad Nacional Agraria La Molina (UNALM) representa cerca de la mitad de las áreas verdes del distrito; siendo el Schinus molle L. «molle serrano» la segunda especie arbórea más abundante en el campus y una de las más plantadas en Lima. Este árbol 
nativo con reconocida importancia cultural es ampliamente utilizado en los arboricultivos ${ }^{1}$ por su carácter ornamental y rústico, resistente a la sequía y plagas; cuya copa conformada por ramas colgantes brinda sombra y al tener una reposición rápida de follaje es capaz de filtrar el aire captando partículas en suspensión y $\mathrm{CO}_{2}$. Sin embargo, se ha observado la presencia de tumoraciones a lo largo del fuste y ramas en los individuos de esta especie alterando su carácter ornamental y representando un probable riesgo. Por lo que se estudió la estructura interna del árbol mediante el tomógrafo forestal Arbosonic 3D y/o la resistencia de la madera sana, madera con tumor y madera con pudrición mediante las propiedades físicas y mecánicas de la madera: densidad básica $(\mathrm{DB})$, resistencia a la flexión o módulo de ruptura (MOR) y rigidez o módulo de elasticidad (MOE). Los resultados determinaron la ausencia de riesgo de caída de árboles de Schinus molle L. por la presencia de tumoraciones, evidenciando la importancia del empleo del tomógrafo forestal en la gestión de los arboricultivos en Lima.

Palabras clave: Schinus molle, tumores, riesgo de caída de árbol, tomografía forestal, arboricultivos.

\section{Is There Schinus molle Tree Risk Due to the presence of burls in the City of Lima?}

\section{Abstract}

La Molina is one of the districts with the bigger green areas and ecological awareness in the city of Lima. The Universidad Nacional Agraria La Molina (UNALM) represents about half of the green areas in the district; being the Schinus molle L. «molle serrano» the second most abundant tree species in the campus and one of the most planted in Lima. This native tree with recognized cultural importance is widely used in the arboriculture crops for its ornamental and rustic character, resistant to the drought and plagues; whose top formed by hanging branches offers shade and when having a fast replacement of foliage, it is able to filter the air capturing particles in suspension and $\mathrm{CO}_{2}$. However, it has been observed the presence of burls along the shaft and branches in trees of this species altering its ornamental character and representing a probable risk. Therefore, the internal structure of the tree was studied using the Arbosonic $3 \mathrm{D}$ forest tomograph and/or the resistance of healthy wood, wood with burl and wood with rot by means of the physical and mechanical properties of the wood: basic density (DB), resistance to bending or modulus of rupture (MOR) and rigidity or modulus of elasticity (MOE). The results determined the absence of Schinus molle L. tree risk due to the presence of burls, evidencing the importance of the use of the forest tomograph in the management of arboriculture crops in Lima.

Keywords: Schinus molle, burls, tree risk, forest tomography, arboricultures.

1 Término referido a la arboricultura.

Def. de arboricultura: ciencia y práctica del cultivo, cuidado y manejo de los árboles y otras plantas leñosas, bien sea como individuos o grupos, por lo regular en lugares urbanos o periurbanos (ISA 2020). 


\section{INTRODUCCIÓN}

Diferentes lugares del mundo consideran importante al arbolado urbano en los paisajes de las ciudades (Mullaney, Lucke y Trueman, 2015; Mira-Martínez, BetancurValencia y Urrego-Giraldo, 2017), principalmente por brindar múltiples beneficios (Goodwin, 2017), como son los relacionados con la regulación ambiental, fines estéticos, valorización de propiedad, y aumento de la calidad de vida del ciudadano (Song, Tan, Edwards y Richards, 2018; FAO, 2016).

El valor que en ellos reside puede ser gestionado bajo una jurisdicción pública o privada (Konijnendijk, Gauthier y Van Veenhuizen, 2005) mediante la planificación, manejo y diseño del arbolado urbano (Pauleit et al., 2002). Es así que los árboles deben encontrarse bajo mantenimiento y evaluación constante; es decir, los gestores y profesionales deben tener experticia y herramientas adecuadas para identificar y disminuir los riesgos en el arbolado urbano hacia las personas y/o sus propiedades (Restrepo, Moreno y Hoyos, 2015).

La Molina es uno de los distritos con mayor cantidad de áreas verdes de la ciudad de Lima (21,5\%) (Municipalidad de La Molina, 2011; Informe Evaluando Lima, 2014) y cuenta con $7,52 \mathrm{~m}^{2}$ de áreas verdes por habitante (SINIA, 2018) valor cercano a los $10 \mathrm{~m}^{2}$ como mínimo recomendado (Lamela, Molini y Salgado, 2011). A su vez, este distrito posee gran conciencia ecológica, por lo que en el 2019 fue elegido parte del proyecto de $\mathrm{APEC}^{2}$ "Ciudades Modelo en Bajas Emisiones de Carbono» (RPP Noticias, 2020). La Universidad Nacional Agraria La Molina (UNALM) abarca aproximadamente el $43 \%$; es decir casi la mitad, de las áreas verdes del distrito (Municipalidad de La Molina, 2011; Informe Evaluando Lima, 2014). El arbolado urbano de la ciudad de Lima - Perú está compuesto por variadas especies forestales, siendo una de las más representativas la especie Schinus molle L. (SERPAR, 2012) que es la segunda más abundante del campus UNALM (Orellana, 2014).

La especie S. molle, conocida como molle serrano, pertenece a la familia Anacardiaceae (Alba, Bonilla y Arroyo, 2009). Original de la zona central y sur de Sudamérica (Rzedowski y Calderón de Rzedowski, 1999), principalmente del Perú (Bailey, 1919). Se distribuye naturalmente desde México hasta América del Sur (Barkley, 1944, 1957; Schulte, Rojas y Rojas, 1992; Crivellaro y Schweingruber, 2013; Bulnes, Orrego y Terán, 2017). Se encuentra en zonas semiáridas y en los valles interandinos, donde es capaz de crecer en áreas bastantes secas, con varios meses sin lluvias. Está ausente en la parte más cálida y húmeda de su área de distribución, y rehúye a las cercanías del mar. Su distribución vertical, según la latitud geográfica, oscila entre los $2000 \mathrm{~m} \mathrm{s.} \mathrm{n.} \mathrm{m.}$

\footnotetext{
2 APEC: Asia-Pacific Economic Cooperation, en español «Foro de Cooperación Económica AsiaPacifico)».
} 
hasta los 3900 metros de altitud (Schulte et al., 1992). Encuentra su mejor desarrollo entre 300 a 620 milímetros de precipitación anual, y en una temperatura media de $19,27{ }^{\circ} \mathrm{C}$ a $21,6{ }^{\circ} \mathrm{C}$ (Conafor, s.f.). Además, se ha cultivado en diferentes partes del mundo (Barkley, 1944), inclusive hasta naturalizarse (Nel et al., 2004).

Estudios realizados por Schulte et al. (1992), Crivellaro y Schweingruber (2013) y Bulnes et al. (2017), acerca de $S$. molle en zonas urbanas y de distribución natural de Lima y Cochabamba respectivamente, lo describen como un árbol ornamental siempre verde de crecimiento moderado a rápido, que alcanza diámetros de 30 a 80 centímetros y alturas entre 6 a 15 metros. La copa es amplia e irregular, compuesta por ramas colgantes. El tronco es sinuoso con corteza externa escamosa con tendencia a desprenderse en placas rígidas de color pardo oscuro a grisáceo. Las raíces son de profundidad media y fasciculada. A comparación de otras especies ornamentales arbóreas, su longevidad, rusticidad, adaptabilidad, bajo requerimiento hídrico y rápido recambio de follaje que permiten la captación de partículas en suspensión y $\mathrm{CO}_{2}$ la convierten en una acertada opción de fácil manejo (Villagrán y Castro, 2004; Nail, 2016; Alvarado, Baldini y Guajardo, 2013).

Este árbol es considerado una especie resistente al ataque de plagas y enfermedades. Tiene como plaga conocida a Calophya schini, "psilido del molle» que le genera agallas en las hojas, lo que provoca su deformación y caída prematura (Móstiga y Lozada, 2019). Por otro lado, Orellana (2014) registró la presencia de tumores en el fuste y ramas de S. molle al interior del campus de la UNALM por primera vez. A pesar de no lograr el aislamiento del agente causal, indicó que la bacteria Agrobacterium tumefaciens podría ser la posible causante de esta anormalidad por la similitud del síntoma presente en especies frutales. De la misma forma Agrios (2005) manifiesta que esa bacteria puede causar tumores en fustes y ramas.

Los tumores son hinchamientos pronunciados anormales en una planta leñosa que, típicamente, ocurren en los troncos y ramas. Se presentan en árboles de todas las edades, y pueden aparecer en un solo árbol o en grupos (Bergdahl y Hill, 2016; Allen et al., 2010). La formación de estos tumores varía en tamaño y generan la deformación de la corteza externa del árbol (Mira-Martínez et al., 2017; Allen et al., 2010). Campbell (2013) y Del Tredici (1999) indican que existen dos tipos de tumores en los árboles: los lignotubérculos, que se encuentran en la parte basal o debajo del suelo y están bajo control genético estricto; y los tumores del tronco, que son una respuesta a condiciones ambientales.

Las causas de las tumoraciones no han sido completamente identificadas, pero se han planteado como posibilidades la inestabilidad genética, el estrés ambiental, las heridas en el cambium por insectos y bacterias, el ataque fúngico, el rocío de agua de mar o la síntesis desregulada de auxina y citoquinina (Eom y Chung, 1994; Allen et al., 2010). 
Los tumores visibles en el exterior del fuste del $S$. molle no solo incurren en una apariencia desordenada y diferente a su fin estético y de ornato urbano, sino que también, podrían dar lugar a variaciones de las condiciones internas que comprometen la estabilidad estructural de árbol y generar un riesgo de caída (Smiley y Fraedrich, 1992; Mattheck y Breloer, 1994). Esta condición puede ser un peligro para la seguridad de la comunidad y la propiedad (Karlinasari, Lestari, Nababan, Siregar y Nandika, 2017).

El análisis de riesgo de caída de árboles puede reducir este riesgo a niveles aceptables, e, inclusive, mantener aquellos árboles viejos, de gran estatura y valiosos en el paisaje urbano (Van Wassenaer y Richardson, 2009), criterio importante a tomar en cuenta en la gestión del arbolado urbano. Este procedimiento utiliza los principios biológicos y de ingeniería para calificar la solidez interna de un árbol, y hacer predicciones razonables del potencial de falla estructural (Wang y Allison, 2008). En Lima, los protocolos a seguir en caso se identifiquen riesgos del arbolado urbano hacia el ciudadano y/o patrimonio se rigen bajo la ordenanza 1852 Ordenanza para la conservación y gestión de áreas verdes para la provincia de Lima (El Peruano, 2014).

La tomografía forestal ha demostrado ser una herramienta muy sensible para detectar, cuantificar y predecir la ubicación de la descomposición de la madera y otros deterioros en el interior del árbol (Wilcox, 1988; Bauer, Kilbertus y Bucur, 1991), estimando su tamaño, forma y características en términos de propiedades mecánicas (Nicolotti, Martinis, Socco, Godio y Sambuelli, 2003). Esta técnica produce una imagen de la estructura transversal interna de un árbol al registrar las diferencias en el tiempo de vuelo (TOF, por sus siglas en inglés) de la onda de impulso ${ }^{3}$ de un sensor transmisor a otro sensor receptor (Karlinasari et al., 2017; Ross, 2015). Si una parte del tronco se dańa y se reduce la densidad de la madera, el TOF es mayor que si el árbol estuviera libre de defectos. Los defectos que son graves reducen el TOF por lo menos en 70\% de los valores típicos de la madera sólida (Bethge, Mattheck y Hunger, 1996).

El uso de la tomografía forestal es ventajoso para el evaluador porque permite generar una vista más completa de las áreas de la sección transversal del fuste y minimiza la probabilidad de daño a las fibras estructural y biológicamente significativas del tronco (Van Wassenaer y Richardson, 2009). No obstante, entre las desventajas que presenta se tiene la falta de precisión para mapear y cuantificar la extensión de los defectos internos (Pokorny, 2003).

Realizar el ensayo de flexión estática para analizar el riesgo de caída de un árbol es una evaluación rigurosa (Costello y Quarles, 1999). Este ensayo permite determinar dos propiedades mecánicas de la madera como son el módulo de ruptura (MOR), que es considerado un criterio de resistencia aceptado y define la capacidad máxima

3 Término que hace referencia a «Sound velocity» (Mattheck y Breloer ,1994), «Stress wave» (Wang y Allison, 2008), «Ultrasonic wave» (Ross, 2015). 
de carga de la madera al doblarse (Kretschmann, 2010); y el módulo de elasticidad (MOE) que es la resistencia de la madera a la deformación bajo una carga aplicada para recuperar sus dimensiones originales cuando esta se elimina (Winandy, 1994). Adicionalmente, es útil conocer la propiedad física de la densidad básica o gravedad específica básica de la madera que se define como la relación masa seca al horno y el volumen en verde (completamente saturado) (Kellogg, 1989; Glass y Zelinka, 2010).

El objetivo de este estudio fue determinar la existencia de un riesgo potencial de caída mediante la evaluación de la alteración de la resistencia natural de la madera con tumor del árbol $S$. molle mediante la tomografía forestal y el estudio de las propiedades físicas y mecánicas, con el fin de generar una base científica para la toma de decisiones en la gestión del arbolado urbano.

\section{IMPORTANCIA DE LA DETERMINACIÓN DEL RIESGO DE CAÍDA}

La presencia del árbol urbano debe ser el contrapunto a la arquitectura de las construcciones en la ciudad, sumando lugares al espacio público que estimulen la vida cultural de los usuarios (González, 2002). Sin embargo, debido a su ubicación geográfica (plazas, avenidas, áreas deportivas y universitarias, etcétera) estos pueden ocasionar perjuicios en los diferentes lugares en donde se ubican, debido a la competencia que existe con el desarrollo urbano (Tovar, 2006). Hay que tener en cuenta que las situaciones de riesgo de los árboles solo se pueden controlar parcialmente ya que, para controlarlos totalmente, se tendría que talar el árbol (Tomao, Secondi, Corona, Giuliarelli, Quantrini y Agrimi, 2015).

Armas, Elías, Espinoza, Figueredo, Hernández, Medina y Ramírez (2008) señalan que realizar un diagnóstico acertado del árbol es fundamental para poder ejecutar un manejo adecuado del problema, de modo que se pueda generar un control efectivo y reducir los efectos negativos, además de generar información técnica y científica. Es así como varios autores han tratado de definir o de conceptualizar la idea de árbol peligroso, y se han tenido presente siempre dos cosas en común: la ubicación geográfica y el potencial de fallo del árbol o de algunas de sus partes (Wallis, Morrison y Ross, 1980; Robbins, 1986; Matheny y Clark, 1994; Albers, Eiber y Hayes, 1996).

Ampliamente se ha usado la evaluación visual del arbolado (VTA, por sus siglas en inglés) (Mattheck, 1994), que consiste en observar las características externas del árbol para deducir las condiciones internas y evaluar la estabilidad estructural (Matheny y Clark, 2009). Sin embargo, De La Vega (2017) reconoce algunas desventajas, entre las cuales afirma que el método está sujeto a la experticia de cada evaluador. De igual manera, se han desarrollado otros métodos basados en observaciones visuales como: el método SIA (Statically Integrated Assessment); el método propuesto por el programa «The Trevey»; entre otros (Calaza, 2007). 
De igual manera, La Municipalidad Metropolitana de Lima (MML), según la ordenanza 1852, determina la peligrosidad de un árbol a través de la observación visual, de modo que se fundamenta la tala y/o poda severa en dos situaciones: si existe un grado de evidente riesgo para la integridad física de bienes o personas, por riesgo muy alto de caída de especie arbórea, y cuando la especie arbórea esté muerta o en muerte regresiva por agentes fitopatológicos u otras causas. En los casos que se tenga que retirar un árbol por emergencia, deberá ser avalado a través de un Informe Técnico de Riesgo (ITR), el cual, debe ser elaborado y suscrito por un profesional (agrónomo, forestal, biólogo o especialidad afín) y comunicado a la MML para ser evaluado por la Subgerencia de Evaluación y Calidad Ambiental de la Gerencia del Ambiente. Si bien es cierto que pueden existir otros mecanismos, este proceso suele ser subjetivo y burocrático algunas veces no permitiendo una acción oportuna.

Sin embargo, la tecnología ha evolucionado desde lo cualitativo a lo cuantitativo haciendo posible cuantificar con mayor precisión la estabilidad y la resistencia a la rotura de un árbol y proporcionar un análisis de riesgo más completo (Van Wassenaer y Richardson, 2009).

\section{Determinación Del Riesgo de CaÍda}

Ningún equipo proporciona una evaluación completa de un árbol (Matheny y Clark, 2009), es por ello que el análisis de riesgo de caída debe considerar otros estudios además de la tomografía forestal (Karlinasari et al., 2017; Wang, Wiedenbeck y Liang, 2009). La madera de los árboles tiene propiedades físicas $(\mathrm{DB})$ y mecánicas $(\mathrm{MOR}$, módulo de ruptura, y MOE, módulo de flexión), cuyos resultados numéricos dan un mayor alcance del estado del individuo en sí, ya que existe una relación estrecha entre lo que se visualiza en un tomograma y la resistencia mecánica del árbol en pie (Ayuntamiento de Madrid, 2014). Las propiedades físicas y mecánicas se pueden predecir con el TOF (Ross, McDonald, Green y Schad, 1997; Ross, 2015; Merlo, Alvarez-Gonzalez, Santaclara y Riesco, 2014). El MOE es la propiedad que está fuertemente correlacionado con el TOF de las ondas de impulso de la tomografía forestal (Ross, 2015).

Se plantea emplear la tomografía forestal y las propiedades físicas y mecánicas de la madera para la determinación de riesgo de caída de los árboles. Ambas pruebas son complementarias y con muestras independientes puesto que la tomografía forestal se realizó a fustes, mientras que la determinación de las propiedades físicas y mecánicas fue de madera de la rama. 


\section{4. ÁREA DE ESTUDIO}

El estudio se realizó en el campus de la Universidad Nacional Agraria La Molina (UNALM), que cuenta con un área de 220 hectáreas aproximadamente, ubicada en el distrito de La Molina (Lima - Perú). Presenta $20^{\circ} \mathrm{C}$ de temperatura anual promedio, $84 \%$ de humedad relativa promedio, y una precipitación anual de 11,9 milímetros (según datos del Observatorio Meteorológico Alexander von Humboldt, UNALM). La topografía está conformada por una terraza de superficie plana, con $1 \%$ de pendiente, drenaje moderado, y permeabilidad y profundidad también moderadas (Alvarez, Ferreyra, Huamantinco y Prieto, 2001). El riego inicialmente fue por gravedad y entre los años 2016 y 2017 cambió a aspersión, cuya instalación conllevó a podas de raíces agresivas en los árboles. Las labores culturales que se han observado en el campus no han sido las adecuadas en algunos casos, como las constantes podas de rejuvenecimiento, el uso de herramientas inapropiadas para las podas como el machete, entre otras. Cabe mencionar que el año en el que se colectaron las muestras y realizaron los estudios (2016 y 2017) hubo fenómeno de El Niño.

\section{Población y muestra}

El campus de la UNALM alberga una población de árboles de S. molle de más de 200 individuos, las tumoraciones en fustes y ramas se presentan en aproximadamente $50 \%$ de los mismos.

\section{Estudio de LA TOMOgRAFía FORESTAL}

\subsection{Muestra}

Los criterios de selección de los árboles para este método fueron: árboles adultos con altura promedio de 6,5 metros y diámetro de altura al pecho (DAP) mayores a $20 \mathrm{~cm}$ - con presencia de tumores en fustes y/o ramas y de edades contemporáneas. En ese sentido, se escogieron tres árboles con tumores, y adicionalmente, un árbol con presencia de algún síntoma o signo de ataque de hongos — esporas en el fuste- Este último caso por ser motivo frecuente de estudios bajo esta técnica.

\subsection{Metodología}

Para el estudio de tomografía forestal se contó con la colaboración del Instituto Nacional de Innovación Agraria (INIA) a través de su personal técnico y el tomógrafo Arbosonic $3 D$, para lo cual se siguió la metodología de Fakopp Enterprise Bt. (2015). Este equipo ha sido diseńado para detectar huecos no visibles, pudriciones o deterioro en el interior 
del fuste del árbol mediante técnicas acústicas no destructivas. La unidad de medición es el tiempo de propagación de la onda de impulso generada por el martillo en el sensor, si existe un hueco en el interior, las ondas lo rodearán, eso significa un aumento del tiempo de recepción por los otros sensores.

El primer paso fue determinar el número y ubicación de las capas a realizarse en el árbol. Las capas de los árboles seleccionados con tumoraciones fueron dos y cuatro, al menos una a la altura del tumor y las otras por encima o por debajo de la excrecencia; mientras que en el árbol seleccionado con presencia de esporas fueron tres, uno a la altura del defecto y las otras dos fueron por encima y por debajo. Se hizo el estudio a este último árbol para realizar una comparación de la estructura interna de un árbol de $S$. molle con un síntoma extensamente estudiado y que es el principal motivo por el cual se producen caídas de árboles, frente a otro síntoma que no se ha estudiado mediante la tomografía forestal. El segundo paso fue determinar la dirección del norte con una brújula, para poder colocar el primer sensor en esa dirección con el martillo de goma. Los siguientes sensores fueron colocados a la misma altura en el fuste (ocho o dieciséis sensores, correlativos a su numeración), en sentido antihorario, y equidistantes entre sí. Después, los sensores se conectaron a las cajas amplificadoras en línea junto con la batería, y la conexión a la tableta (el ordenador) se dio mediante Bluetooth. Finalmente, los cabezales de los sensores fueron golpeados tres veces con un martillo de acero, cuyos datos se registraron en el ordenador. Estos pasos se repitieron en las diferentes capas determinadas para cada árbol.

El software instalado en el ordenador se encarga de recopilar los datos de tiempo mediante Bluetooth, obtener el tomograma de la sección transversal interna del árbol y permitir la visualización individual o en conjunto de las capas de un individuo.

La tomografía forestal con el equipo Arborsonic 3D tiene una escala de coloración donde verde define la madera sana o intacta; a medida que el grado de color disminuye a más claro pasando por amarillo hasta llegar a rojo, refleja una madera con pudrición; y cuando la coloración se torna celeste, quiere decir que existe un hueco o ausencia de madera (Figura 1). La evaluación de los tomogramas se realizó sobre la base de las tres categorías propuestas por Karlinasari et al. (2017) para otro equipo de tomografía, pero con el mismo principio de ondas de esfuerzo. Inicialmente se debe calcular con el software el porcentaje de madera intacta en cada tomograma de un árbol, luego seleccionar el porcentaje más bajo para ese individuo. Finalmente, se procedió a categorizar ese valor, si el porcentaje de madera intacta es mayor a $80 \%$ le corresponde la categoría de árbol saludable, si el porcentaje de madera intacta está comprendida entre $50 \%$ y $80 \%$ la categoría es moderadamente saludable y si es menor a $50 \%$ la categoría es árbol con el tronco poco saludable. 
Figura 1. Escala de coloración para el tomógrafo Arbosonic 3D

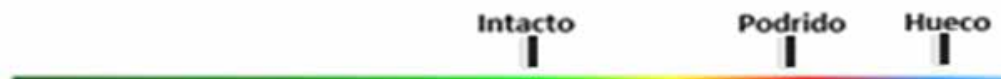

Fuente: Fakopp Enterprise Bt. (2015).

Para el mejor entendimiento de la tomografía, previamente al uso del tomógrafo, se elaboró unos bosquejos de la distribución de los tumores y otras particularidades en la circunferencia del fuste donde se realizaron las tomografías, para analizar la diferencia entre lo observado a simple vista y lo que ocurre en el interior del árbol. La leyenda del bosquejo solamente considera tumores, esporas y cicatrices de poda.

Figura 2. Leyenda de los bosquejos de la circunferencia del fuste

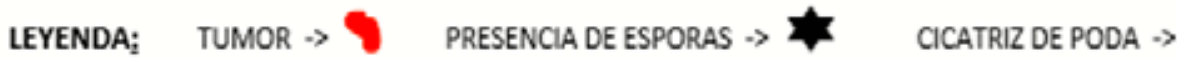

\subsection{Análisis interpretativo}

Los resultados de los bosquejos y los tomogramas realizados a los individuos se relacionaron en un análisis interpretativo mediante el cual, se puede establecer comparaciones entre lo observado en campo y la estructura interna del tronco.

\subsubsection{Resultados y discusiones de la tomografía forestal}

La tomografía forestal de los cuatro árboles de $S$. molle se realizaron en diferentes alturas y considerando diferentes cantidades de tomogramas y de sensores (Tabla 1).

Tabla 1. Mediciones y cantidad de sensores para la tomografía forestal

\begin{tabular}{cccccc}
\hline $\mathbf{N}^{\circ}$ árbol & Tomograma & Código & Altura (m) & $\begin{array}{c}\text { Circunferencia/ } \\
\text { Diámetro (cm) }\end{array}$ & $\mathbf{N}^{\circ}$ sensores \\
\hline 1 & 1 & $1 \mathrm{~A} 1$ & 0,60 & $284 / 90$ & 16 \\
1 & 2 & $1 \mathrm{~A} 2$ & 1,50 & $372 / 118$ & 16 \\
2 & 1 & $2 \mathrm{~A} 1$ & 0,00 & $218 / 69$ & 8 \\
2 & 2 & $2 \mathrm{~A} 2$ & 0,90 & $95 / 30$ & 8 \\
3 & 1 & $3 \mathrm{~A} 1$ & 0,20 & $136 / 43$ & 8 \\
3 & 2 & $3 \mathrm{~A} 2$ & 0,80 & $111 / 35$ & 8 \\
3 & 3 & $3 \mathrm{~A} 3$ & 1,40 & $120 / 38$ & 8 \\
\hline
\end{tabular}




\begin{tabular}{cccccc}
\hline $\mathbf{N}^{\circ}$ árbol & Tomograma & Código & Altura (m) & $\begin{array}{c}\text { Circunferencia/ } \\
\text { Diámetro (cm) }\end{array}$ & $\mathbf{N}^{\circ}$ sensores \\
\hline 4 & 2 & $4 \mathrm{~A} 2$ & 1,10 & $99,5 / 32$ & 8 \\
4 & 3 & $4 \mathrm{~A} 3$ & 1,70 & $100 / 32$ & 8 \\
4 & 4 & $4 \mathrm{~A} 4$ & 1,86 & $87,5 / 28$ & 8 \\
\hline
\end{tabular}

En la Figura 3, el árbol 1 tiene dos tomogramas y sus pares en bosquejos. El tomograma $1 \mathrm{~A} 1$ no muestra ningún tipo de afectación en la madera, concordando con su bosquejo 1B1. El tomograma 1A2, en contraste con el tomograma anterior, se corresponde con el bosquejo 1B2 que presenta tumores en 50\% del perímetro aproximadamente, pero no manifiesta madera afectada. Como consecuencia de ello, los tomogramas $1 \mathrm{C}$ y $1 \mathrm{D}$ en 3D (tres dimensiones) no exponen ningún defecto interno en el fuste de este árbol, aunque presenta tumoraciones de grandes dimensiones. Este tipo de representaciones en 3D sirve para mejorar la ejecución de métodos no destructivos posteriores que evalúan la resistencia de la madera de árboles en pie como el penetrómetro (Allison y Wang, 2015).

Figura 3. Tomografía forestal del árbol 1 de Schinus molle
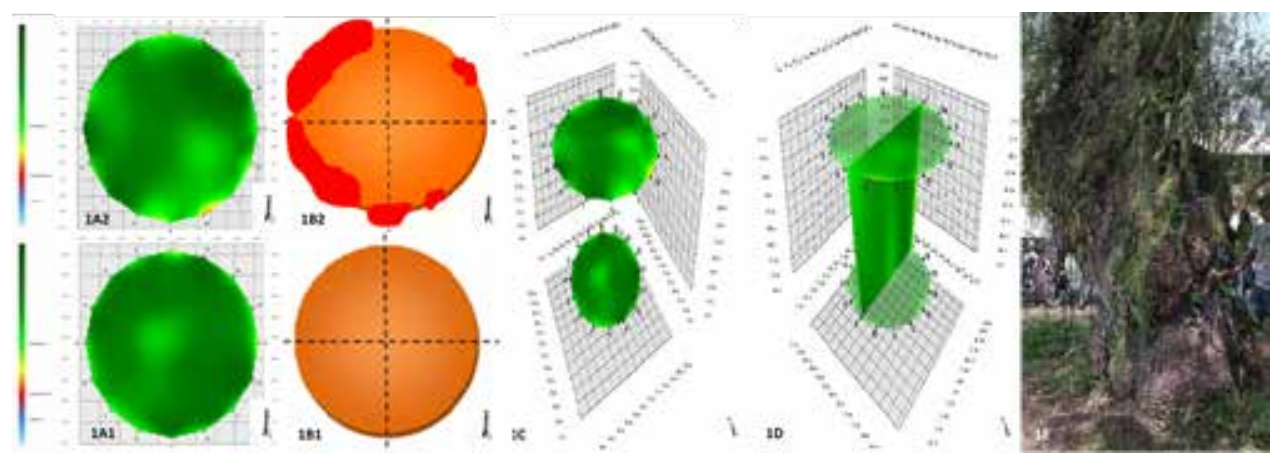

*1A1 y $1 \mathrm{~A} 2$ son tomogramas en $2 \mathrm{D} .1 \mathrm{~B} 1$ y $1 \mathrm{~B} 2$ son bosquejos. 1C y $1 \mathrm{D}$ son proyecciones de los tomogramas en 3D. 1E es una fotografía del fuste del árbol

En la Figura 4, el árbol 2 también posee dos tomogramas y dos bosquejos. El tomograma $2 \mathrm{~A} 1$ muestra madera intacta, mientras que el bosquejo $2 \mathrm{~B} 1$ refleja la presencia de tumores en el perímetro del fuste. El tomograma 2A2, también presenta el 100\% de madera intacta y el bosquejo 2B2 solo presenta una cicatriz de poda. Debido a estos tomogramas, las respectivas proyecciones $2 \mathrm{C}$ y $2 \mathrm{D}$ no muestran madera diferente a la intacta. 


\section{Figura 4. Tomografía forestal del árbol 2 de Schinus molle}
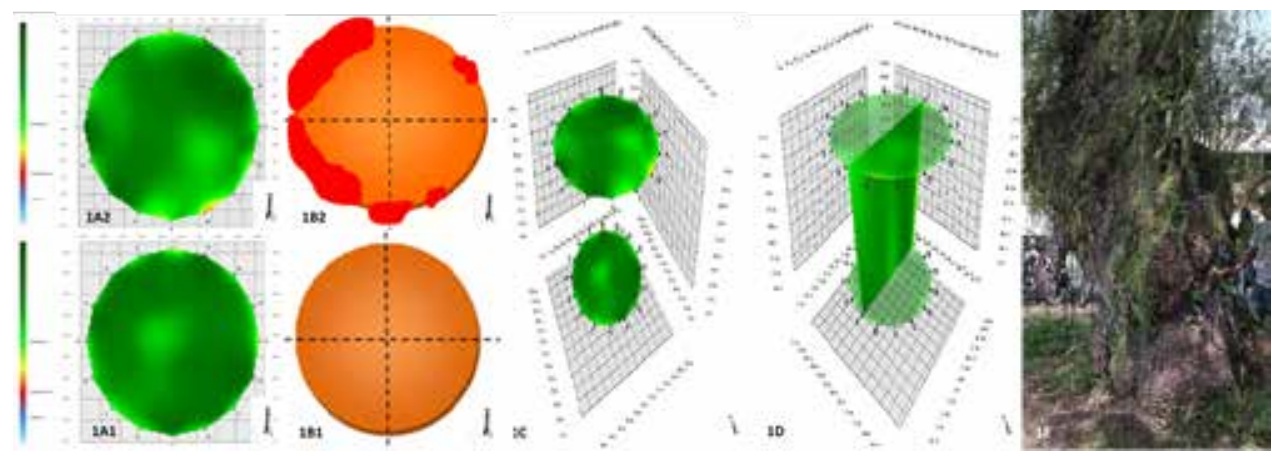

*2A1 y $2 \mathrm{~A} 2$ son tomogramas en $2 \mathrm{D}$. $2 \mathrm{~B} 1$ y $2 \mathrm{~B} 2$ son bosquejos. $2 \mathrm{C}$ y $2 \mathrm{D}$ son proyecciones de los tomogramas en 3D. 2E es una fotografía del fuste del árbol.

En la Figura 5, al árbol 3 se le realizaron tres tomogramas y tres bosquejos. El tomograma 3A1 evidencia sólo madera intacta y el bosquejo 3B1 muestra dos cicatrices de poda. El tomograma $3 \mathrm{~A} 2$ revela madera con pudrición y una cavidad en la zona noreste del tronco, mientras que el bosquejo 3B2 indica la presencia de esporas en la zona norte del perímetro del fuste. El tomograma $3 \mathrm{~A} 3$ muestra un aspecto similar al anterior; sin embargo, el bosquejo 3B3 no presenta ninguna afectación en el exterior. No se puede considerar el área del defecto en los tomogramas como preciso, ya que el tomograma muestra el defecto más grande que la descomposición real. La tomografía forestal confirma la presencia del defecto, pero no es precisa porque no diferencia entre la descomposición y las grietas (Wang, Allison, Wang y Ross, 2007; Wang y Allison, 2008; Lin y Yang, 2015). En ese sentido, las proyecciones 3C y 3D, resultantes de la combinación de los tomogramas, muestran la magnitud y el avance de la pudrición a lo largo del fuste.

En la Figura 6, el árbol 4, a diferencia de los otros árboles, tiene cuatro tomogramas y cuatro bosquejos. El tomograma $4 \mathrm{~A} 1$ muestra un $5 \%$ de pudrición en la zona oeste; sin embargo, el bosquejo 4B1 no indica presencia de esporas ni de tumor en esa zona. El tomograma 4A2 muestra una pequeña porción de madera de transición de intacta a pudrición, mientras que el bosquejo $4 \mathrm{~B} 2$ solo presenta un tumor en una zona diferente. El tomograma $4 \mathrm{~A} 3$ tiene la misma característica que el anterior, aunque el bosquejo $4 \mathrm{~B} 3$ presenta tumores en el $50 \%$ del perímetro y una cicatriz de poda. El tomograma 4A4 también presenta una porción de madera de transición, pero el bosquejo $4 \mathrm{~B} 4$ no manifiestan anormalidades. Estas tonalidades cerca al perímetro y entre los sensores son indicativos de deterioro; sin embargo, estos no son reales porque al constatar con los bosquejos, no existe ninguna anormalidad en la zona en cuestión. Según Wu, Li, Jiao y Wang (2018), estos falsos positivos son un problema común en los tomogramas. Por consiguiente, se puede resolver que los tomogramas $4 \mathrm{C}$ y $4 \mathrm{D}$ reflejan un fuste saludable. 
Figura 5. Tomografía forestal del árbol 3 de Schinus molle

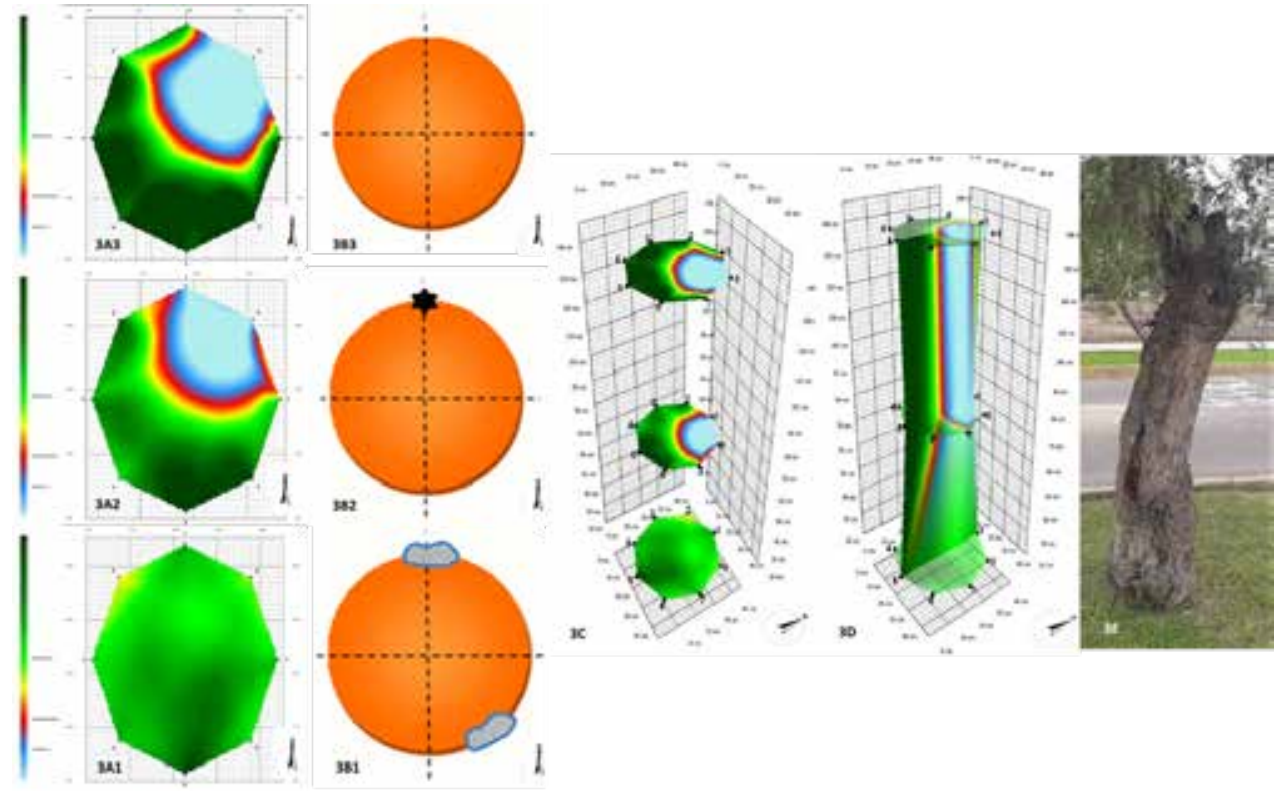

*3A1 y $3 \mathrm{~A} 2$ son tomogramas en 2D. 3B1 y $3 \mathrm{~B} 2$ son bosquejos. 3C y 3D son proyecciones de los tomogramas en 3D. 3E es una fotografía del fuste del árbol.

Figura 6. Tomografía forestal del árbol 4 de Schinus molle
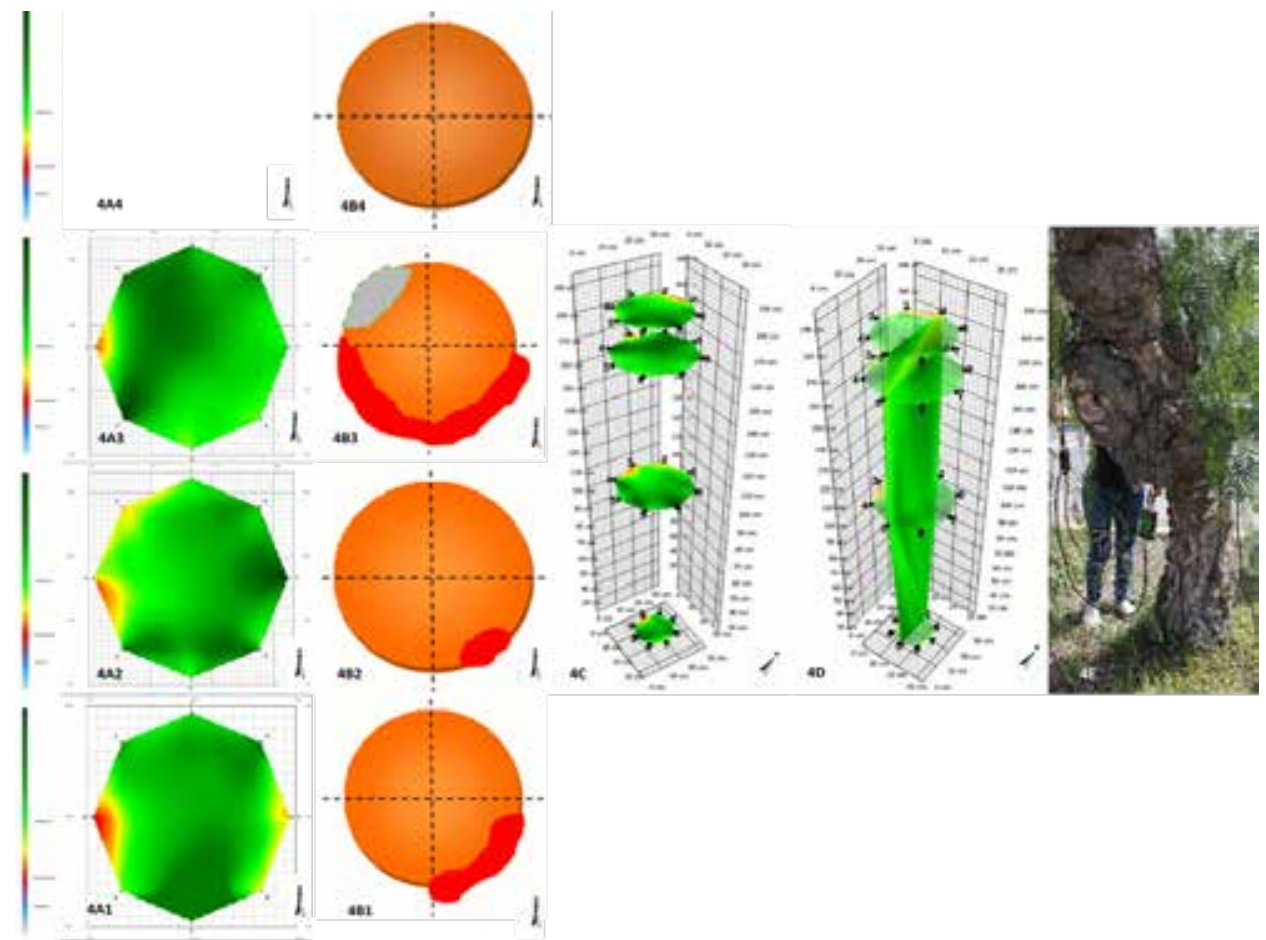

*4A1 y $4 \mathrm{~A} 2$ son tomogramas en $2 \mathrm{D} .4 \mathrm{~B} 1$ y $4 \mathrm{~B} 2$ son bosquejos. $4 \mathrm{C}$ y $4 \mathrm{D}$ son proyecciones de los tomogramas en 3D. 4E es una fotografía del fuste del árbol. 
La interpretación de los tomogramas clasifica a los árboles con tumores en la categoría de árbol saludable porque tienen un porcentaje de madera intacta o sólida mayor al $80 \%$, mientras que el árbol con pudrición se encuentra en la categoría moderada puesto que el porcentaje de madera intacta está entre 50\% y $80 \%$ (Tabla 2).

Tabla 2. Interpretación de los tomogramas en porcentajes

\begin{tabular}{cc}
\hline Código & \multicolumn{1}{c}{ Interpretación del tomograma } \\
\hline 1A1 & Intacta: 100\%; Pudrición: 0\%; Hueco: 0\% \\
1A2 & Intacta: 100\%; Pudrición: 0\%; Hueco: 0\% \\
2A1 & Intacta: 100\%; Pudrición: 0\%; Hueco: 0\% \\
2A2 & Intacta: 100\%; Pudrición: 0\%; Hueco: 0\% \\
3A1 & Intacta: 100\%; Pudrición: 0\%; Hueco: 0\% \\
3A2 & Intacta: 68\%; Pudrición: 5\%; Hueco: 27\% \\
3A3 & Intacta: 52\%; Pudrición: 5\%; Hueco: 43\% \\
4A1 & Intacta: 95\%; Pudrición: 5\%; Hueco: 0\% \\
4A2 & Intacta: 100\%; Pudrición: 0\%; Hueco: 0\% \\
4A3 & Intacta: 100\%: Pudrición: 0\%;Hueco: 0\% \\
4A4 & Intacta: 100\%; Pudrición: 0\%; Hueco: 0\% \\
\hline
\end{tabular}

Cabe mencionar que no existe la posibilidad de que los factores que afectan a las ondas de impulso, como el contenido de humedad, el diámetro del árbol y la temperatura de operación (Wang, 2013), hayan sesgado los resultados de la tomografía forestal. El contenido de humedad no ha podido afectar el estudio, debido a que todas las tomografías se tomaron en árboles vivos, donde la madera está en condición saturada. Del mismo modo, el diámetro del árbol no ha podido influir puesto que los diámetros de los árboles evaluados se encuentran dentro de los rangos aceptables para no atenuar las ondas de impulso como sí lo hacen los de grandes diámetros (Pokorny, 2003). Respecto a la temperatura de operación, tampoco ha influido, ya que la temperatura promedio ambiental de La Molina es de $20^{\circ} \mathrm{C}$, la cual está entre $0{ }^{\circ} \mathrm{C}$ y $30^{\circ} \mathrm{C}$ donde las ondas de impulso son constantes (Gao, Wang, Wang y Allison, 2013).

Considerando lo mencionado por Orellana (2014) acerca de que Agrobacterium tumefaciens es el posible agente causal de los tumores en S. molle, Agrios (2005) indica que las bacterias de este género no generan cavidades internas en las plantas. Asimismo, al comparar los bosquejos con los tomogramas se puede afirmar que las tumoraciones no modifican lo suficiente a las propiedades de la madera como para ser detectadas por la tomografía forestal, en cambio, las pudriciones y los huecos ocasionados por hongos si son posibles de encontrar. De igual manera, los tomogramas en 3D, para el 
caso de los árboles con tumores, proyectan la condición de madera intacta a lo largo del fuste, de forma contrapuesta, el tomograma en 3D del árbol con pudrición muestra el avance longitudinal de la degradación de la madera y del hoyo.

Por otro lado, el Ayuntamiento de Madrid (2014) realizó un estudio de riesgo de caída de árboles en el Parque del Retiro en España, por medio del uso del tomógrafo para determinar la existencia de pudrición y su intensidad. Esta investigación ha encontrado una relación estrecha entre los resultados del tomógrafo que muestra la parte interna del individuo y la resistencia natural del árbol en pie, las cuales se han visto poco afectadas por la presencia de tumores. Es posible que la madera con tumor no genere cambios en el color de la tomografía forestal, porque una reducción en la elasticidad de la madera (tumoración) aumentará la velocidad del sonido, a diferencia de una reducción en la densidad de la madera (pudrición) causará su disminución (Pokorny, 2003; Ross, 2015; Allison y Wang, 2015).

\section{ESTUDIO DE LAS PROPIEDADES FÍSICAS Y MECÁNICAS DE LA MADERA}

\subsection{Muestra}

Se obtuvieron dos tipos de probetas: «probetas de madera con tumor» provenientes de árboles con tumoraciones y "probetas de madera sana» (testigo) provenientes de árboles sin tumoraciones.

Los criterios de selección de árboles para la obtención de probetas para este estudio fueron: ramas primarias de árboles de edades contemporáneas, con necesidad de poda, DAP (diámetro a la altura del pecho) promedio de $40 \mathrm{~cm}$ y altura promedio de $6,5 \mathrm{~m}$, con presencia de tumores en ramas y fustes para obtener las "probetas de madera con tumor» y sin presencia de ellos para las "probetas de madera sana». Es preciso señalar que todas las probetas se obtuvieron a partir de ramas. Asimismo, es importante mencionar que algunas "probetas de madera con tumor» contenían algún porcentaje de madera libre de tumor; mientras que las "probetas de madera sana» se obtuvieron de árboles sin tumoración.

\subsection{Metodología}

El estudio de las propiedades físicas y mecánicas de la madera se llevó a cabo en el Laboratorio de Tecnología de la Madera del Departamento Académico de Industrias Forestales (DAIF) de la Facultad de Ciencias Forestales (FCF) de la UNALM y se utilizaron probetas de madera en condición verde, con la finalidad de simular la reacción del árbol en pie al resistir una carga. La propiedad física estudiada fue la densidad básica y las propiedades mecánicas fueron el módulo de ruptura (MOR) y de elasticidad (MOE). 


\section{a. Determinación de la propiedad física: densidad básica (DB)}

Para la determinación de la densidad básica se tuvo como base la Norma Panamericana COPANT 461. Las dimensiones de las probetas de madera con tumor fueron reducidas a $1,5 \mathrm{~cm} \times 1,5 \mathrm{~cm} \times 1,5 \mathrm{~cm}$ debido a la falta de material maderable y a que se quería obtener un número de muestras aceptables.

b. Determinación de las propiedades mecánica: módulo de ruptura (MOR) y módulo de elasticidad (MOE)

Para la determinación del módulo de ruptura (MOR) y módulo de elasticidad (MOE) se llevó a cabo una serie de ensayos de flexión estática. Dichas pruebas se realizaron en la prensa universal Tinus Olsen con una frecuencia de $8,4 \mathrm{~cm} / \mathrm{min}$ aplicada para la especie, según la Norma Técnica Peruana (NTP) 251.017 (ITINTEC, 1980).

\subsection{Análisis estadístico}

El análisis estadístico de las propiedades mecánicas y físicas de la madera se realizó mediante el programa MINITAB 18. El cumplimiento de supuestos de normalidad de errores se verificó con la prueba Kolmogórov-Smirnov (también llamada prueba K-S) y la de homogeneidad de varianzas con la prueba F de Fisher. La comparación de las medias se realizó a través de la prueba de T de Student.

\subsection{Resultados y discusiones de las propiedades físicas y mecánicas de la madera}

\section{a. Densidad básica (DB)}

La densidad básica (DB) de la madera sana de $S$. molle fue de $0,417 \mathrm{~g} / \mathrm{cm}^{3} \pm 37,5 \mathrm{~g} /$ $\mathrm{cm}^{3}$. Este valor está por debajo a la $\mathrm{DB}$ de la madera proveniente de un árbol dentro de su distribución natural $\left(0,556 \mathrm{~g} / \mathrm{cm}^{3}\right)$ y al de un árbol introducido en una zona urbana $\left(0,500 \mathrm{~g} / \mathrm{cm}^{3}\right)$ (Schulte et al., 1992; Crivellaro y Schweingruber, 2013). Cabe precisar que la madera utilizada para este estudio provino de una rama que puede tener igual o mayor densidad básica que la madera de fuste (Dadzie, Amoah, Frimpong-Mensah e Inkum, 2016; Okai, 2002). La madera con tumor presentó una densidad básica $\left(0,394 \mathrm{~g} / \mathrm{cm}^{3} \pm 28,3 \mathrm{~g} / \mathrm{cm}^{3}\right)$ igual al de la madera sana estadísticamente (Tabla 3). Por otro lado, Fallas, Holbrook, Rocha, Vásquez y Gutiérrez (2010) encontraron que la madera de tumor tiene una densidad básica inferior respecto a la madera sana de la especie Cochlospermum vitifolium (Bixaceae); sin embargo, Govorčin, Sinković, Sedlar, Šefc e Ištok (2012), El Mouridi et al. (2011), Tsoumis, Kezos, Fanariotou, Voulgaridis y Passialis (1988), Gülsoy, Eroğlu y Merev (2005) señalan que la madera con tumor es ligeramente mayor que la normal para las especies Erica arborea (Ericaceae), Tetraclinis articulata (Cupressaceae), Picea glauca (Pinaceae), Quercus robur subsp. robur (Fagaceae). Esta diferencia se debe a que la madera con tumor está compuesta por crecimientos 
excesivos oscuros, de alta densidad, que son células con paredes más gruesas incrustadas en una matriz de tejido leñoso, de densidad cercana a la madera sana (El Mouridi et al., 2011). Es posible que la madera con tumor se constituya de una proporción mayor de parénquima axial y menor de fibras, para servir al árbol como almacenamiento de agua, nutrientes y carbohidratos, aunque esta característica sea propia de tumores basales o lignotubérculos (Bamber y Mullette, 1978; James, 1984).

Tabla 3. Densidad básica de la madera sana y de tumor de Schinus molle

\begin{tabular}{lc}
\hline Propiedad física de la madera & $\mathrm{DB}\left(\mathrm{g} / \mathrm{cm}^{3}\right)$ \\
\hline Madera sana & $417,1 \pm 37,5^{\mathrm{A} 1}$ \\
Madera con tumor & $394,1 \pm 28,3^{\mathrm{A}}$ \\
\hline${ }^{1}$ Promedio de 10 repeticiones; promedios en una columna con letras diferentes \\
son significativamente diferentes a un nivel de probabilidad del $5 \%$.
\end{tabular}

\section{b. Módulo de ruptura (MOR) y módulo de elasticidad (MOE)}

Como se indica en la tabla 4, la madera sana obtuvo valores de MOR $\left(241,17 \mathrm{~kg} / \mathrm{cm}^{2}\right.$ $\left.\pm 63,98 \mathrm{~kg} / \mathrm{cm}^{2}\right)$ y MOE $\left(89019,56 \mathrm{~kg} / \mathrm{cm}^{2} \pm 29708,78 \mathrm{~kg} / \mathrm{cm}^{2}\right)$ superiores a las de la madera con tumor (MOR: 177,00 kg/ $\mathrm{cm}^{2} \pm 69,98 \mathrm{~kg} / \mathrm{cm}^{2}$; MOE: $67459,71 \mathrm{~kg} / \mathrm{cm}^{2}$ $\pm 26860,93 \mathrm{~kg} / \mathrm{cm}^{2}$ ); es decir, es más resistente y rígida. Esto se evidenció puesto que la probeta de madera con tumor demoró entre diez y quince minutos menos en romperse. De otra manera, El Alami et al. (2013) encontró mayor resistencia; es decir un MOR superior, en la madera con tumor que en la normal para la especie Tetraclinis articulata (Cupressaceae).

La madera con tumor tiene todos los elementos celulares que la madera sana (Chattaway, 1958), aunque presenta una estructura anatómica diferente (El Mouridi et al., 2011). En otras palabras, la orientación de las células de la madera con tumor está desorganizada (Gülsoy et al., 2005), dispuesta en forma de contorta (Paula, Naulin, Arce, Galaz y Pausas, 2016). Por todo ello, las propiedades mecánicas de la madera con tumor son notoriamente diferentes de la madera sana (Rosero, Hernández y Riedl, 2017).

MAE y FAO (2014) indican que el MOR de la madera de S. molle es $745,43 \mathrm{~kg} / \mathrm{cm}^{2}$. Este valor es mucho mayor que el obtenido para la madera sana, lo cual se explica por el uso de probetas saturadas provenientes de las ramas. El empleo de probetas saturadas implica que las propiedades mecánicas de la madera se incrementen por el contenido de humedad por debajo del punto de saturación de las fibras (Gerhards, 1982). Asimismo, se estima que los valores de MOR y MOE de probetas provenientes de ramas puedan ser iguales o mayores que las de fuste (Dadzie et al., 2016; Amoah, Appiah-Yeboahand y Okai, 2012; Okai, 2002). 
Finalmente, cabe señalar que las probetas de madera con tumor al no estar constituidas enteramente por madera con tumor generan una mayor variabilidad en los datos de resistencia y elasticidad (El Alami et al., 2013).

Tabla 4. Módulo de ruptura (MOR) y módulo de elasticidad (MOE) de la madera sana y de tumor de Schinus molle

\begin{tabular}{lcc}
\hline Propiedades mecánicas de la madera & MOR $\left.\mathrm{kg} / \mathrm{cm}^{2}\right)$ & MOE $\left(\mathrm{kg} / \mathrm{cm}^{2}\right)$ \\
\hline Madera sana & $241,17 \pm 63,98^{\mathrm{A} 1}$ & $89019,56 \pm 29708,78^{\mathrm{A}}$ \\
Madera con tumor & $177,00 \pm 69,98^{\mathrm{B}}$ & $67459,71 \pm 26860,93^{\mathrm{B}}$ \\
\hline
\end{tabular}

${ }^{1}$ Promedio de 33 repeticiones; promedios en una columna con letras diferentes son significativamente diferentes a un nivel de probabilidad del $5 \%$.

\section{RECOMENDACIONES}

Se recomienda la evaluación periódica del estado físico (arquitectura e inclinación del árbol) y fitosanitario, y el mantenimiento preventivo de los árboles de Schinus molle L. con ausencia o presencia de tumores mediante la poda. Este tratamiento debe realizarse con la técnica y en la época apropiada. Asimismo, las podas deben evitar que las ramas se excedan en peso sin alterar la forma natural de la copa propia de la especie. También se recomienda realizar investigaciones para determinar el agente causal de las tumoraciones.

La tomografía arbórea debe ser una herramienta prioritaria para la toma de decisiones en la extracción de árboles con riesgo de caída en la ciudad.

\section{Conclusiones}

La tomografía forestal y las propiedades físicas y mecánicas de la madera evidencian la ausencia de riesgo de caída de árboles de Schinus molle L. por la presencia de tumoraciones; siendo la tomografía forestal una técnica importante para la gestión de los arboricultivos en Lima y pudiendo estar acompañado de estudios destructivos en etapas de investigación.

\section{Agradecimiento}

Al Vicerrectorado de Investigación y al Vicerrectorado Académico de la UNALM por el financiamiento otorgado. A la Facultad de Ciencias Forestales (FCF) y al Laboratorio de Tecnología de la Madera de la UNALM, al Mg. Sc. Moisés Acevedo Mallque por permitirnos utilizar sus instalaciones y equipos y al Sr. Vicente Cuba Huamán, técnico del Laboratorio. También al Instituto Nacional de Innovación Agraria (INIA), 
a su director de la Sub-Dirección de Investigación y Estudios Especiales el doctor José Eloy Cuellar Bautista (2007-2019), a las ingenieras Haydeé Miriam Ramos León y Haru García Mesa, y a Víctor Ayala Lara por el apoyo técnico en la tomografía forestal. Finalmente, al Mg. Sc. Rolando Montenegro por su apoyo en el procesamiento estadístico.

\section{REFERENCIAS}

Agrios, G. (2005). Plant pathology (Quinta ed.). Amsterdam, Holanda: Elsevier Academic Press.

Alba, A., Bonilla, P. y Arroyo, J. (2009). Actividad cicatrizante de una pomada con aceite esencial de Schinus molle L. «Molle» en ganado vacuno con heridas infectadas y en ratones. Ciencia e Investigación, 12(1), 29-36.

Albers, J., Eiber, T. y Hayes, E. (1996). How to recognize hazardous defects in trees. U.S. Department of Agriculture, Forest Service. Recuperado de https://vtcommunityforestry.org/sites/default/files/pictures/how_to_recognize_hazardous_defects_in_trees.pdf

Allen, K., Blodgett, J., Burns, K., Cain, R., Costello, S., Eager, T.,... Worral, J. (2010). Field guide to diseases \& insects of the Rocky Mountain region. Fort Collins, CO: U.S. Department of Agriculture, Forest Service. Recuperado de https://www.fs.usda.gov/ Internet/FSE_DOCUMENTS/stelprdb5262952.pdf

Allison, R. y Wang, X. (2015). Nondestructive testing in the urban forest. En R. Ross, Nondestructive evaluation of wood (pp. 77-86). Madison, WI: U.S. Department of Agriculture, Forest Service, Forest Products Laboratory.

Alvarado, A., Baldini, A. y Guajardo, B. (2013). Arboles urbanos de Chile: Guia de Reconocimiento. Santiago: Corporación Nacional Forestal. Recuperado de https://www. conaf.cl/cms/editorweb/institucional/Arboles_urbanos_de_Chile-2da_edicion.pdf

Alvarez, G., Ferreyra, M., Huamantinco, B. y Prieto, R. (2001). Diagnóstico de la gestión integral y propuesta de desarrollo para el Jardín Botánico «Octavio Velarde Núñez» de la Universidad Nacional Agraria La Molina (trabajo de suficiencia profesional). Universidad Nacional Agraria La Molina, Lima, Perú.

Amoah, M., Appiah-Yeboahand, J. y Okai, R. (2012). Characterization of physical and mechanical properties of branch, stem and root wood of Iroko and Emire tropical trees. Research Journal of Applied Sciences, Engineering and Technology, 4(12), 1755-1761.

Armas, Y., Elías, R., Espinoza, L., Figueredo, E., Hernández, L., Medina, R. y Ramírez, M. (2008). El diagnóstico y su papel en la fitoprotección. Temas de Ciencia y Tecnología, 12(35), $47-54$.

Ayuntamiento de Madrid. (2014). Informe fisico-mecánico mediante análisis instrumental del arbolado en el Parque del Retiro. Madrid, España. Recuperado de http://www.madrid. es/UnidadesDescentralizadas/LimpiezaUrbanaYResiduos/Descriptivos/ficheros/ PrimerInformedelGrupodeExpertosdelArbolado.pdf 
Bamber, R. y Mullette, K. (1978). Studies of the lignotubers of Eucalyptus gummifera (Gaertn. and Hochr.) II. Anatomy. Australian Journal of Botany, 26(1), 15-22. https:// doi.org/10.1071/BT9780015

Barkley, F. (1944). Schinus L. Brittonia, 5(2), 160-198.

Barkley, F. (1957). A study of Schinus L. Lilloa(28), 5-110.

Bauer, C., Kilbertus, G. y Bucur, V. (1991). Technique ultrasonore de caractérisation du degré d'altération des bois de hêtre et de pin soumis à l'attaque de différents champignons. Holzforschung, 45(1), 41-46. https://doi.org/10.1515/hfsg.1991.45.1.41

Bailey, L. (1919). Schinus. Standard Cyclopedia of Horticulture. https://doi.org/10.5962/ bhl.title.23351

Bergdahl, A. y Hill, A. (2016). Diseases of trees in the Great Plains. Fort Collins, CO: U.S. Department of Agriculture, Forest Service. Recuperado de https://www.fs.usda.gov/ nac/documents/research/publications/rmrs_gtr335.pdf

Bethge, K., Mattheck, C. y Hunger, E. (1996). Equipment for detection and evaluation of incipient decay in trees. Journal of Arboriculture, 20, 13-37. https://doi.org/10.10 80/03071375.1996.9747095

Bulnes, F., Orrego, M. y Terán, A. (2017). Árboles y palmeras del vivero forestal: caracteristicas, requerimientos y recomendaciones de 40 especies para la arboricultura urbana. Lima, Perú: Jesús G. Bellido M.

Calaza, P. (2007). Revisión bibliográfica y análisis comparativo de métodos de evaluación de riesgo de arbolado urbano. Caso particular: La Coruña (tesis doctoral). Universidad de Santiago de Compostela, Galicia, España. Recuperado de http://hdl.handle. net/10347/2324

Campbell, R. (2013). Exploring the mysteries of redwood burls. Recuperado de https:// www.savetheredwoods.org/blog/wonders/exploring-the-mysteries-of-redwood-burls/

Chattaway, M. (1958). Bud development and lignotuber formation in eucalypts. Australian Journal of Botany, 6, 103-115. https://doi.org/10.1071/BT9580103

Costello, L. y Quarles, S. (1999). Detection of wood decay in blue gum and elm: An evaluation of the Resistograph and the portable drill. Journal of Arboriculture, 25(6), 311-317. Recuperado de http://unri.org/ECO\%20697U\%20S14/costello_quarles,_1999.pdf

Comisión Nacional Forestal (CONAFOR). (s.f.). Schinus molle L. SIRE: CONABIOPRONARE Recuperado de http://www.conafor.gob.mx/portal/docs/secciones/ reforestacion/Fichas\%20Tecnicas/Schinus\%20molle.pdf

Comisión Panamericana de Normas Técnicas (COPANT). (1972). Método de determinación del peso específico aparente. Norma Panamericana COPANT 461. Lima, Perú.

Crivellaro, A. y Schweingruber, F. (2013). Atlas of wood, bark and pith anatomy of eastern mediterranean trees and shrubs: with a special focus on Cyprus. Nueva York, NY: Springer Science \& Business Media. https://doi.org/10.1007/978-3-642-37235-3 
Dadzie, P., Amoah, M., Frimpong-Mensah, K. y Inkum, P. (2016). Variation in bending strength properties in stem and branch woods as influenced by density and moisture levels in Entandrophragma cylindricum. Australian Forestry, 79(4), 233-240. https:// doi.org/10.1080/00049158.2016.1251816

De La Vega, F. (2017). Descripción y análisis de la metodología de evaluación visual de árboles urbanos - VTA (vissual tree assessment) (trabajo monográfico). Universidad Nacional Agraria La Molina, Lima, Perú. Recuperado de http://repositorio.lamolina. edu.pe/bitstream/handle/UNALM/3441/de-la-vega-centuri\%C3\%B3n-fernando. pdf? sequence $=1 \&$ isAllowed $=y$

Del Tredici, P. (1999). Redwood burls: immortality underground. Arnoldia, 59, 14-22.

El Alami, S., El Mouridi, M., Laurent, T., Calchéra, G., Famiri, A., Hakam, A.,... Gril, J. (2013). Fracture energy of Wood and root burl Wood of thuya (Tetraclinis articulata). Journal of Tropical Forest Science, 25(2), 166-174.

El Mouridi, M., Laurent, T., Famiri, A., Kabouchi, B., Alméras, T., Calchéra, G.,... Hakam, A. (2011). Caractérisation physique du bois de la loupe de thuya (Tetraclinis articulata (Vahl) Masters). Physical and Chemical News, 59, 57-64. https://doi.org/hal-00797386

El Peruano. (18 de diciembre de 2014). Ordenanza N 1852: Ordenanza para la conservación y gestión de áreas verdes en la provincia de Lima. Lima, Perú.

Eom, Y. y Chung, Y. (1994). Tumor Wood anatomy in korean red pine (Pinus densiflora). International Association of Wood Anatomists Journal, 15(2), 149-155. https://doi. org/10.1163/22941932-90001354

Fallas, L., Holbrook, M., Rocha, O., Vásquez, N. y Gutiérrez, M. (2010). Phenology, lignotubers, and water relations of Cochlospermum Vitifolium, a pioneer tropical dry forest tree in Costa Rica. Biotropica, 42(1), 104-111. https://doi.org/10.1111/j.17447429.2009.00539.x

Fakopp Enterprise Bt. (2015). Arbosonic 3D: Manual del usuario. Ágfalva, Hungría: s.e.

Food and Agriculture Organization of the United Nations (FAO). (2016). Beneficios de los árboles urbanos. Recuperado de http://www.fao.org/resources/infographics/ infographics-details/es/c/411598/

Gao, S., Wang, X., Wang, L. y Allison, R. (2013). Effect of temperature on acoustic evaluation of standing trees and logs: part 2 -field investigation. Wood and Fiber Science, 45(1), 15-25.

Gerhards, C. (1982). Effect of moisture content and temperature on the mechanical properties of wood: An analysis of immediate effects. Wood and Fiber, 14(1), 4-36.

Glass, S. y Zelinka, S. (2010). Moisture relations and physical properties of Wood. En F. P. Laboratory, Wood handbook: wood as an engineering material (pp. 4-1-4-19). Madison, WI: U.S. Department of Agriculture, Forest Service, Forest Products Laboratory.

Goodwin, D. (2017). Urban tree. Londres, Reino Unido: Routledge. https://doi. org/10.4324/9781315266169 
Gonzales, C. (2002). Beneficios del arbolado urbano (ensayo doctorado). Recuperado de https://digital.csic.es/bitstream/10261/24578/1/Beneficios\%20del\%20arbolado\%20 urbano.pdf

Govorčin, S., Sinković, T., Sedlar, T., Šefc, B. y Ištok, I. (2012). Properties of trunk and briarwood of three heth (Erica arbórea L.) from island Rab. En R. Németh y A. Teischinger, The 5th conference on hardwood research and utilisation in Europe 2012 (pp. 71-78). Sopron, Hungría: University of West Hungary Press.

Gülsoy, S., Eroğlu, H. y Merev, N. (2005). Chemical and Wood anatomical properties of tumorous Wood in a turkish White oak (Quercus robur subsp. robur). International Association of Wood Anatomists Journal, 26(4), 469-476. https://doi. org/10.1163/22941932-90000128

Informe Evaluando Lima. (2014). Cómo vamos en ambiente. Recuperado de http://www. limacomovamos.org/cm/wp-content/uploads/2015/10/ReporteAmbiente2014_virtual.pdf

Instituto de Investigación Tecnológica Industrial y de Normas Técnicas, Perú (ITINTEC). 1980. Método de ensayo de flexión estática. NTP 251.017. Lima, Perú.

International Society of Arboriculture (ISA). (02 de junio del 2020). ISA Dictionary online. Recuperado de https://wwv.isa-arbor.com/education/onlineresources/dictionary.

James, S. (1984). Lignotubers and burls: Their structure, function and ecological significance in Mediterranean ecosystems. The Botanical Review, 50(3), 225-266. https:// doi.org/10.1007/BF02862633

Karlinasari, L., Lestari, A., Nababan, M., Siregar, I. y Nandika, D. (2017). Assessment of urban tree condition using Sonic tomography technology. IOP Conference Series Earth and Environmental Science, 203, 12-30. https://doi.org/10.1088/1755$1315 / 203 / 1 / 012030$

Kellogg, R. (1989). Density and porosity. En A. Schniewind, Concise encyclopedia of Wood \& wood-based materials (pp. 79-82). Cambridge, MA: Pergamon Press, Oxford/MIT Press.

Kretschmann, D. (2010). Mechanical properties of wood. En F. P. Laboratory, Wood handbook: wood as an engineering material (pp. 5-1-5-44). Madison, WI: U.S. Departament of Agriculture, Forest Service, Forest Products Laboratory. Recuperado de https:// www.fpl.fs.fed.us/documnts/fplgtr/fpl_gtr190.pdf

Konijnendijk, C., Gauthier, M. y Van Veenhuizen, R. (2005) Árboles y ciudades-Creciendo juntos. Revista Agricultura Urbana, 13. Recuperado de https://ruaf.org/assets/2020/01/ RAU13.pdf

Lamela, A., Molini, F. y Salgado, M. (2011). En búsqueda de unas recomendaciones urbanísticas mundiales de densidad y espacios verdes. Nimbus, 27-28, 95-118.

Lin, C. y Yang, T.-H. (2015). Detection of acoustic velocity and electrical resistance tomographies for evaluation of peripheral-inner wood demarcation in urban royal 
palms. Urban Forestry \& Urban Greening, 14, 583-589. https://doi.org/10.1016/j. ufug.2015.05.010

Matheny, N. y Clark J. (1994). A photographic guide to the evaluation of hazard trees in urban areas. Second edition. Savoy, IL: International Society of Arboriculture.

Matheny, N. y Clark, J. (2009). Tree risk assessment. What we know? (and What we don't know?). Arborist News, 18, 28-33. Recuperado de https://www.researchgate. net/publication/292089898/download

Mattheck, C. y Breloer, H. (1994). Field guide for visual assessment. Arboricultural Journal, 18(1), 1-23. https://doi.org/10.1080/03071375.1994.9746995

Merlo, E., Alvarez-Gonzalez, J., Santaclara, O. y Riesco, G. (2014). Modelling modulus of elasticity of Pinus pinaster Ait. in northwestern Spain with standing tree acoustic measurements, tree, stand and site variables. Forests Systems, 23, 153-166. https:// doi.org/10.5424/fs/2014231-04706

Ministerio del Ambiente del Ecuador (MAE) y Organización de las Naciones Unidas para la Alimentación y la Agricultura (FAO). (2014). Propiedades anatómicas, fisicas y mecánicas de 93 especies forestales. Recuperado de http://www.fao.org/3/a-i4407s.pdf

Mira-Martínez, J., Betancur-Valencia, S. y Urrego-Giraldo, L. (2017). Relación entre la infección por agallas, las variables estructurales y la anatomía de la madera de Rhizophora mangle L., en el golfo de Urabá (Colombia). Actualidades Biológicas, 39(106), 41-52. https://doi.org/10.17533/udea.acbi.v39n106a04

Móstiga, M. y Lozada, P. (2019). Insectos y ácaros perjudiciales en los arboricultivos de la Universidad Nacional Agraria La Molina, Perú. IDESIA, 37(1), 115-124.

Mullaney, J., Lucke, T. y Trueman, S. (2015). A review of benefits and challenges in growing street trees in paved urban environments. Landscape and Urban Planning, 134, 157-166. https://doi.org/10.1016/j.landurbplan.2014.10.013

Municipalidad de La Molina. (junio, 2011). Áreas verdes por vecino. Recuperado de http:// www.munimolina.gob.pe/index.php/la-molina/areas-verdes-por-vecino

Nail, S. (2016). Cambio climático: lecciones de y para ciudades de América Latina. Bogotá, Colombia: Universidad Externado de Colombia.

Nel, J., Richardson, D., Rouget, M., Mgidi, T., Mdzeke, N., Le Maitre, D.,... Neser, S. (2004). A proposed classification of invasive alien plant species in South Africa: towards prioritising species and areas for management action. South Africa Journal of Science, 100, 53-64.

Nicolotti, R., Martinis, R., Socco L.V., Godio A. y Sambuelli, L. (2003). Application and comparison of three tomographic techniques for detection of decay in trees. Recuperado de https://www.researchgate.net/publication/242619380_Application_and_comparison_of_three_tomographic_techniques_for_detection_of_decay_in_trees

Okai, R. (2002). A study on the milling and strength properties of branchwood. International Timber Tropical Organization Tropical Forest Update, 12, 24-25. 
Orellana, G. (2014). Prospección y evaluación de sintomas y signos de enfermedades en especies forestales del campus de la Universidad Nacional Agraria La Molina (tesis de pregrado inédita). Universidad Nacional Agraria La Molina, Lima, Perú. Recuperado de http:// repositorio.lamolina.edu.pe/handle/UNALM/880

Paula, S., Naulin, P., Arce, C., Galaz, C. y Pausas, J. (2016). Lignotubers in Mediterranean basin plants. Plant Ecology, 217, 661-676. https://doi.org/10.1007/s11258-0150538-9

Pauleit, S., Jones, N., García-Marín, G., García-Valdecantos, J., Rivière, L., Vidal-Beaudet, L. Bodson, M. y Randrup, T. B. (2002). Tree stablishment practice in towns and cities - results from a European survey. Urban Forestry \& Urban Greening, 1(2), 83-96. https://doi.org/ 10.1078/1618-8667-00009

Pokorny, J. (2003). Urban Tree Risk Management: A Community Guide to Program Design and Implementation. Saint Paul, MN: U.S. Departament of Agriculture. Recuperado de https://www.fs.usda.gov/naspf/sites/default/files/publications/02_na-tp-0303_urban_tree_risk_management_508c_v2_20171027.pdf

Restrepo, H., Moreno, F. y Hoyos, C. (2015). Incidencia del deterioro progresivo del arbolado urbano en el Valle de Aburrá Colombia. Colombia Forestal, 18(2), 225-240. https://doi.org/ 10.14483/udistrital.jour.colomb.for.2015.2.a04

Robbins, K. (1986). How to recognize and reduce tree hazards in recreation sites: USDA Forest Service, Northeastern Area. NA-FR-31. Recuperado de https://archive.org/ details/CAT10286730/page/n1/mode/2up

Rosero, J., Hernández, R. y Riedl, B. (2017). Effects of Fire-retardant Treatment and Burl Wood Structure on Three-dimensional Changes of Sandwich Panels Made from Walnut Decorative Veneer. BioResources, 12(3), 6471-6489. https://doi.org/10.15376/ biores.12.3.6471-6489

Ross, R. (2015). Static bending, transverse vibration, and longitudinal stress wave nondestructive evaluation methods. En R. Ross, Nondestructive evaluation of wood (pp. 5-20). Madison, WI: U.S. Department of Agriculture, Forest Service, Forest Products Laboratory.

Ross, R., McDonald, K., Green, D. y Schad, K. (1997). Relationship between log and timber modulus of elasticity. Forest Products Journal, 47(2), 89-92.

RPP Noticias. (24 de febrero de 2020). Dos distritos del Perú son modelo para la reducción de emisión de CO2. Recuperado de https://rpp.pe/peru/lima/distritos-del-peru-sonmodelo-para-la-reduccion-de-emision-de-co2-noticia-1247582?ref=rpp

Rzedowski, J. y Calderón de Rzedowski, G. (1999). Anacardiaceae. Flora del Bajio y de regiones adyacentes. Fascículo 78. Pátzcuaro, Michoacán, México: Instituto de EcologíaCentro Regional del Bajío. Consejo Nacional de Ciencia y Tecnología y Comisión Nacional para el Conocimiento y Uso de la Biodiversidad. 
Schulte, A., Rojas, C. y Rojas, R. (1992). Reforestación y agroforestería en los andes: uso sostenido, conservación y restauración de suelos con árboles y arbustos nativos: apuntes sobre el molle (Schinus molle L.). Cochabamba: ETSFOR, FUPAGEMA, AGRUCO, ECO. Recuperado de http://atlas.umss.edu.bo:8080/xmlui/handle/123456789/43

Servicio de Parques de Lima (SERPAR). (2012). Árboles de Lima. Lima, Perú: Walter H. Wust.

Sistema Nacional de Información Ambiental (SINIA). (2018). Indicador: Superficie de área verde urbana por habitante en Lima Metropolitana. Recuperado de https://sinia. minam.gob.pe/indicador/998

Smiley, E. y Fraedrich, B. (1992). Determining strength loss from decay. Journal of Arboriculture, 18(4), 201-204.

Song, X., Tan, P., Edwards, P. y Richards, D. (2018). The economic benefits and costs of trees in urban forest stewardship: A systematic review. Forestry \& Urban Greening, 29, 162-170. https://doi.org/10.1016/j.ufug.2017.11.017

Tomao, A., Secondi, A., Corona, P., Giuliarelli, D., Quantrini, V. y Agrimi, M. (2015). Can composite indices explain multidimensionality of tree risk assessment? A case study in an historical monumental complex. Urban Forest \& Urban Greening, 14(3), 456-465. https://doi.org/ https://doi.org/10.1016/j. ufug.2015.04.009

Tovar, G. (2006). Manejo del arbolado urbano en Bogotá. Colombia Forestal, 9(19), $187-$ 205.

Tsoumis, G., Kezos, N., Fanariotou, I., Voulgaridis, E. y Passialis, C. (1988). Characteristics of briarwood. Holzforschung, 42(2), 71-77. https://doi.org/10.1515/hfsg.1988.42.2.71

Van Wassenaer, P. y Richardson, M. (2009). A review of tree risk assessment using minimally invasive technologies and two case studies. Arboricultural Journal, 32, 275-292. https://doi.org/10.1080/03071375.2009.9747583

Villagrán, C. y Castro, V. (2004). Ciencia indigena de los Andes del norte de Chile. Santiago, Chile: Editorial Universitaria.

Wallis, G., Morrison, D. y Ross, D. (1980). Tree hazards in Recreation Sites in Columbia. B.C. Ministry of Lands, Parks and Housing. Canadian Forestry Service. Joint Report 13.

Wang, X. (2013). Acoustic measurements on trees and logs: a review and analysis. Wood Science and Technology, 47, 965-975. https://doi.org/10.1007/s00226-013-0552-9

Wang, X. y Allison, R. (2008). Decay detection in red oak trees using a combination of visual inspection, acoustic testing, and resistance microdrilling. Arboriculture and Urban Forestry, 34(1), 1-4. Recuperado de https://www.fpl.fs.fed.us/documnts/ pdf2008/fpl_2008_wang001.pdf

Wang, X., Allison, R., Wang, L. y Ross, R. (2007). Acoustic tomography for decay detection in red oak trees. Madison, WI: U.S. Department of Agriculture, Forest Service, Forest Products Laboratory. https://doi.org/10.2737/FPL-RP-642 
Wang, X., Wiedenbeck, J. y Liang, S. (2009). Acoustic tomography for decay detection in black cherry trees. Wood and Fiber Science, 41(2), 127-137.

Wilcox, W. (1988). Detection of early stages of wood decay with ultrasonic pulse velocity. Forest Products Journal, 38(5), 68-73.

Winandy, J. (1994). Wood properties. En C. Amtzen, Encyclopedia of Agricultural Science (pp. 549-561). Orlando, FL: Academic Press. Recuperado de https://www.fpl.fs.fed. us/documnts/pdf1994/winan94a.pdf

Wu, X., Li, G., Jiao, Z. y Wang, X. (2018). Reliability of acoustic tomography and groundpenetrating radar for tree decay detection. Applications in Plant Sciences, 6(10), e1 187. https://doi.org/10.1002/aps3.1187 